\title{
Genetic Evolution and Self-Organization of Cellular Robotic System*
}

\author{
Yoshio KAWAUCHI**, Makoto INABA** \\ and Toshio FUKUDA***
}

\begin{abstract}
A cellular robotic system (CEBOT) is a system composed of many kinds of units called "cells". A cell has a simple function and limited intelligence. In task executions, many cells are required to carry out a same task cooperatively to supplement their functions each other. If some cells have malfunctions, the total performance of the system can maintained by exchanging the malfunctioning cells. Therefore, CEBOT is expected to be adaptable to any kinds of tasks and environments. It is important to study self-organization and self-evolution of CEBOT, because both the hardware and software of CEBOT consist of many kinds of cells which are autonomous agents as mentioned above. This paper addresses self-organization of the hardware and selfevolution of the software based on the Genetic Algorithm. An outline of cells developed by the authors is reported. A self-evolutional knowledge base addressed in this paper is based on the Genetic Algorithm. The self-evolutional knowledge base is applied to a simple task planning system installed into a manipulator system and is verified. The effectiveness of the knowledge base is demonstrated by showing a learning capability of the manipulator in experiments.
\end{abstract}

Key Words : Genetic Algorithm, Self-Organization, Evolution, Multi-Agent System, Distributed Autonomous System, Task Planning, Mechatronics and Robotics

\section{Introduction}

As one of the way to use a system to execute many kinds of tasks under different circumstances, a system has been proposed which is divided into simple elements with functions and knowledge hereinafter called cells and which dynamically regroups these cells. This system has been proposed and reported under the name of Cellular Robotic System (CEBOT), and is similar to structures of living organisms in that it builds a complicated system by grouping simple elements and that this build-up takes place in a selforganizing fashion (Fig. 1)(1)-(7). In order for CEBOT to execute unknown tasks under unknown circumstances without human assistance, it is desirable that

* Received 1st December, 1993. Japanese original: Trans. Jpn. Soc. Mech. Eng., Vol. 58, No. 556, C (1992) pp. 3667-3674. (Received 5th March, 1992)

** Applied Technology Dept., Toyo Engineering Corporation, 2-8-1 Akanehama, Narashino-shi, Chiba 275, Japan

*** Dept. of Mechano-Informatics and Systems, Nagoya University, Furo-cho, Chikusa-ku, Nagoya 464-01, Japan

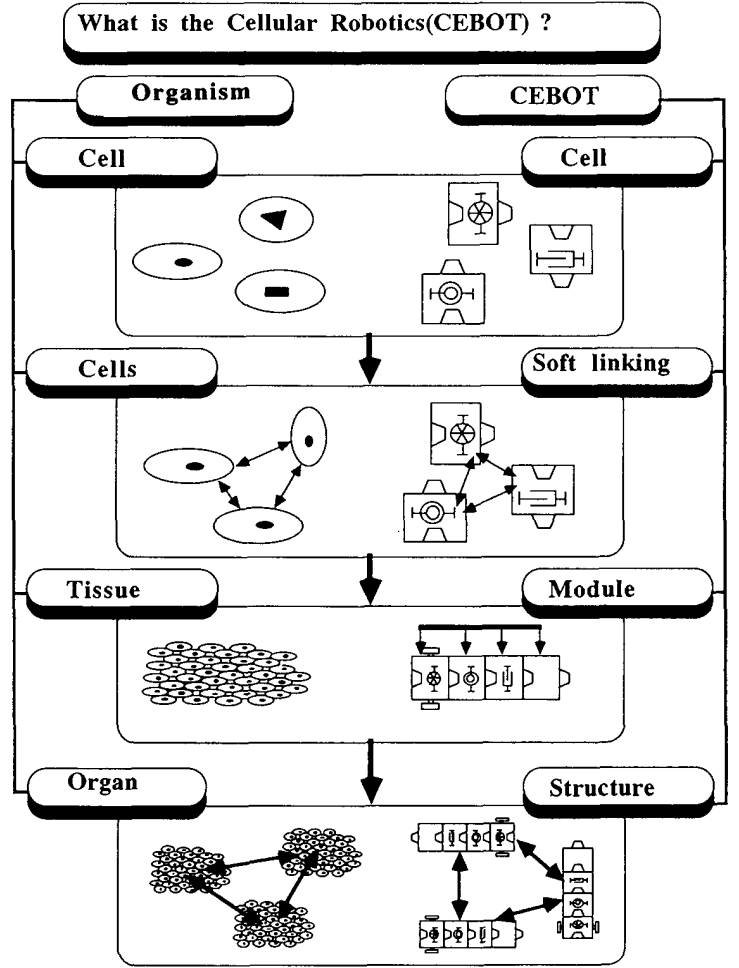

Fig. 1 Concept of CEBOT 
it is not only a dynamically reconfigurable robot but also self-evolving. This paper reports on the selforganization and self-evolution of CEBOT. Because a great variety of species evolve in the world of living organisms, only those which can adapt to the environment, survive. This paper proposes an algorithm called genetic cell production algorithm which enable knowledge system to self-organize evolutionally. The genetic cell production algorithm is based on a principle of natural selection modeled by genetic algorithms. With respect to a task planning system composed of a group of knowledge elements called knowledgecells, this algorithm was applied to processes of generating and eliminating of the knowledge-cells appropriate to environments and tasks in the similar way that living tissues are possessed. Moreover, a prototype robot manipulator has been configurated, and operates unknown objects by using proposed task planning system. The effectiveness of the proposed task planning system is demonstrated by experimental results of the manipulator system.

\section{Concept of Cellular Robotic System (CEBOT)}

CEBOT uses an approach similar to that of biological systems. The idea is to have a few very simple components which are able to connect and separate freely. In this way a flexible dynamically changeable structure is realizable. Many cells can connect to modules, and many modules can interact to create very complex systems. CEBOT has many unique features similar to these of biological organisms. Selfredundancy on the cell level can be achieved by replacing malfunctioning components or by repairing faults using end-effector cells included in the entire CEBOT. If repair or replacement is not possible, other system modules or components can substitute for the function of defective parts. The flexible hardware structure of CEBOT, as well as its software system is freely reconfigurable.

A single component of CEBOT, referred to as a "cell", has its own intelligence, self-contained software, independent data base, sensor system, communication capabilities, a coupling mechanism, and a single mechanical function. Cells are classified in to 3 groups according to their mechanical function:

Group 1: Active actuating cells such as bending joint cells, rotating joint cells, sliding joint cells, end-effector cells, and mobile cells (wheel, crawler, etc.).

Group 2: Branching cells, orientation-changing cells, length-adjustable arm cells between joints, power cells for heavy-duty work, etc.

Group 3: Work cells, end-effector cells, and specialpurpose cells.
Cell connection and separation are carried out automatically by mobile cells interacting with other cells. Joint and end-effector cells, which are already combined to form a manipulator, can attach or detach other cells.

Like biological organisms, CEBOT is also organized in a hierarchically structured system (see Figs. 1 and 2). In the lowest level (LEVEL 5 shown in Fig. 2 ), we find cells with the basic functions of groups 2 , 3. These cells have their own independent CPU, selfcontained software, data base, and sensor systems. They are principally completely autonomous intelligent units. However, basic cells of LEVEL 5 are not able to move actively, which distinguishes them from the mobile or joint cells of group 1. These active cells are placed in LEVEL 4 . Cells of LEVELs 4 and 5 can freely communicate with each other. Cells in LEVEL 4 are masters of modules, which they form together with several LEVEL 5 cells. When cells build up modules or larger structures, the module master searches for its partner cells and automatically approaches and docks with them.

Several modules can combine to form large structures, a process that is controlled by the coordination level (LEVEL 3). In many applications the coordination of uncoupled modules or mobile cells is desirable, and is also controlled in LEVEL 3.

The structure planning level (LEVEL 2) generates structures depending on given tasks. The required number of cells, the cell types, and their combination sequences are decided.

Input commands to the structure planning level arise from the task planning level (LEVEL 1), which is the highest level, and corresponds to the central nervous system of living organisms.

\section{Self-Organizing Hardware}

\section{1 Hardware configuration of a cell}

The authors have developed several prototypes of

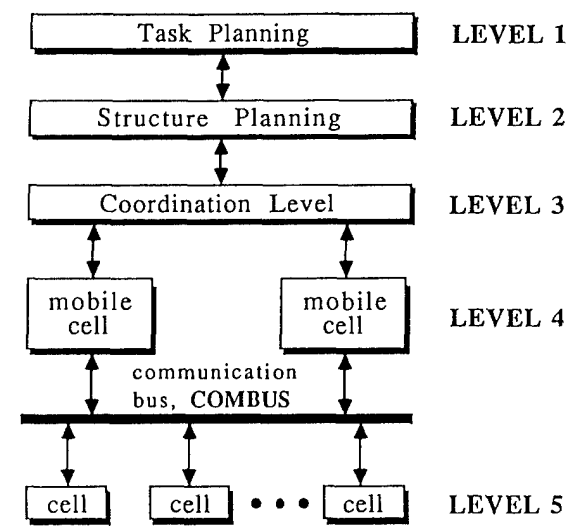

Fig. 2 Hierarchical system architecture of CEBOT 
CEBOT. Figure 3 shows the appearance of a mobile cell of CEBOT Mark II. The mobile cell has two wheels controlled by stepping motors. Each cell has sensors for cell recognition (infrared sensor), position and attitude detection (infrared sensor) and obstacle avoidance (ultrasonic sensor). For close distance position and attitude finding $(0-0.5 \mathrm{~m})$, eight infrared LEDs on a cell and three infrared photo-diodes in the mobile cell are used. The eight LEDs face eight different directions at 45 degrees intervals (Fig. 4 (a)). One ultrasonic transmitter and two receivers are used for obstacle detection (Fig. 4(b)). This sensor arrangement can recognize obstacles at distance as far as $0.7(\mathrm{~m})$ and inclination angles up to 30 degrees.

Each cell has two communication devices. One is used for communication with separated cells, the other is a communication system based on a busline laid in cells for communication with connected cells.

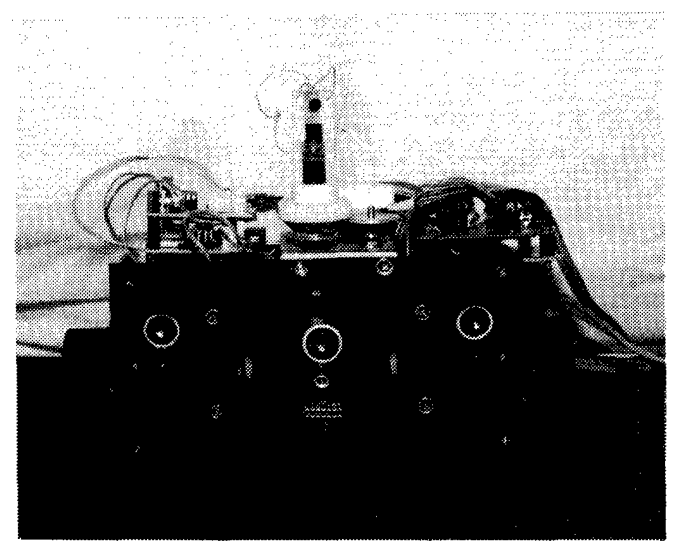

Fig. 3 Appearance of a mobile cell (Mark II)

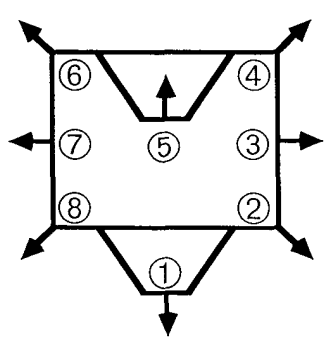

Fig. 4 (a) Eight LEDs for position and orientation recognition

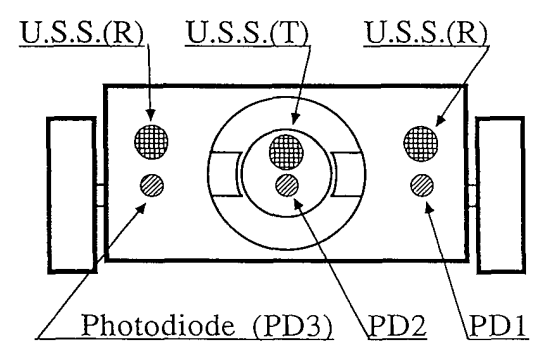

Fig. 4 (b) Photo diodes and ultrasonic sensors

\section{2 Automatic approach and docking method}

Automatic docking has been realized by two cells without telecommunication based on the proposed communication protocol ${ }^{(2),(7)}$. If more than two cells exist in a system, it is necessary for them to communicate with each other for cell identification and position and orientation recognition. The communication realizes more efficiently autonomous docking than the docking without communication among cells. Communication is achieved by an infrared LED/photo diode pair mounted in a rotating sensor module on an elevated level (Fig. 3). Signal transfer to the LED/ photo diode is done by four brush and slip-ring sets. The mobile cell calls for a docking partner with the desired cell function, eventually selects it out of several cells of the same type, and locates its position. Based on the information a master cell obtains, the cells can identify a target cell and dock it and separate autonomously from the cell ${ }^{(7)}$. Figure 5 shows the appearance of a module which is composed of autonomously docked cells. CEBOT has the ability of hardware self-organization by using mobile cells, connection mechanisms, and communication systems $^{(7)}$.

\section{Self-Organizing Software}

\section{1 Model of self-organizing system}

As an example of a self-organizing system, a task planning system having the self-evolving and selforganizing capabilities is proposed in this paper. As shown in Fig. 6, this system is composed primarily of two parts, namely a group of elements called knowledge-cells and a data base. Knowledge-cell group is composed of a set of basic units called knowledgecells to be defined later, and stratified as shown in Fig. 7.

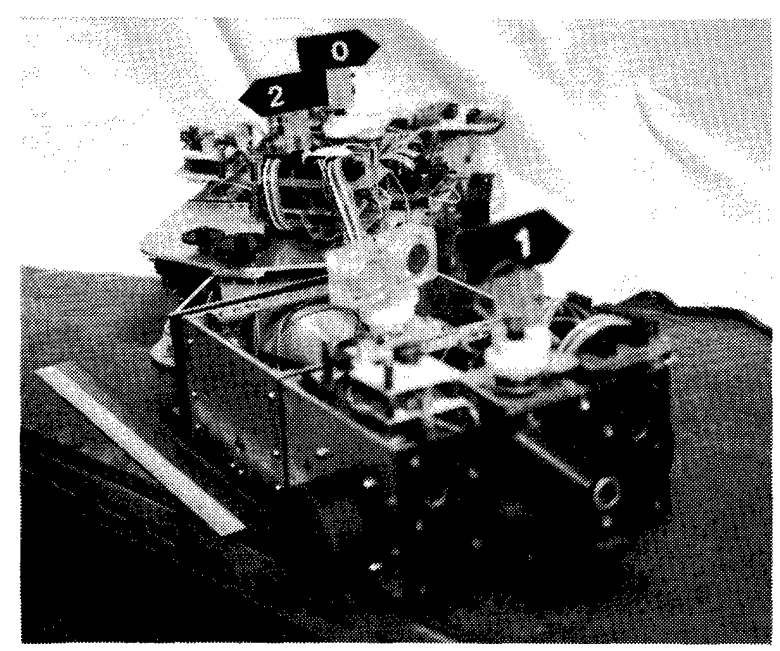

Fig. 5 Appearance of a module composed of five cells (Mark II) 


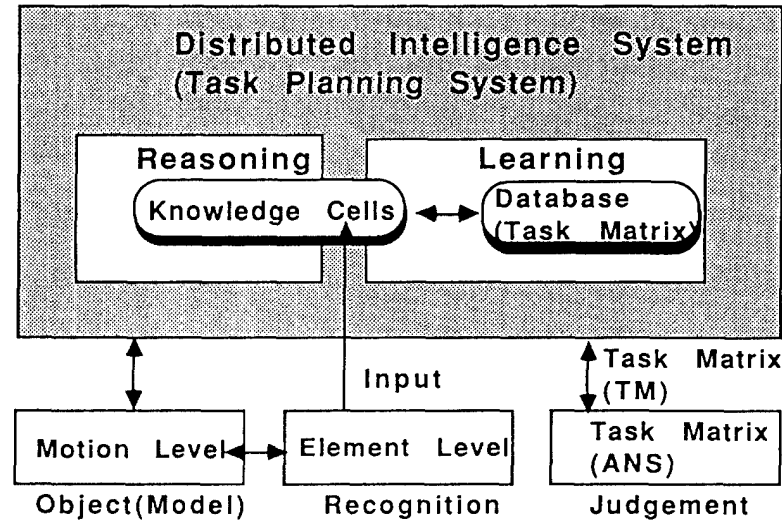

Fig. 6 Task planning system having cell structure

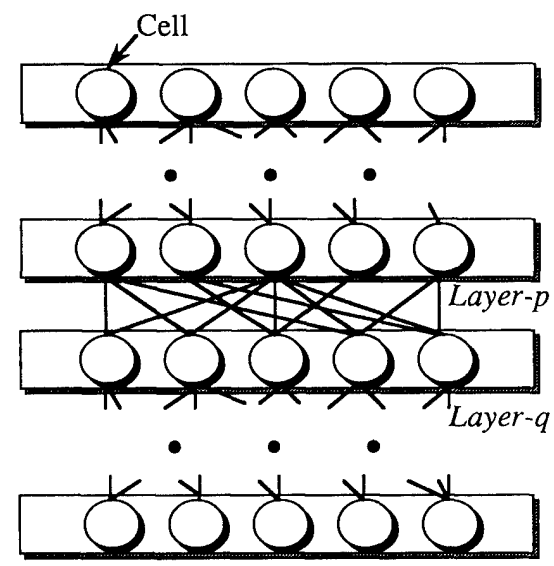

Fig. 7 Hierarchical structure of knowledge cell

Knowledge-cell : basic unit of the system. It connects with other knowledge-cells and has its own knowledge and capability of learning. Positive numbers called connection weights are assigned to connections between knowledge cells. The normalized connection weight is called connection intensity. The normalization means that the sum of all the connection weights on the connection line of the knowledge cell is 1 . A vector which has connection intensity as its element is defined as the knowledge possessed by that knowledge-cell (Fig. 8).

Data base : knowledge possessed by a knowledgecell is used for search and estimation of which cells among a number of stratified knowledge--cells should be used, and each knowledge-cell stores knowledge for procedural actions and various data which cannot be described by its connection intensity, and accesses to the database whenever the cell is activated by a certain task. This database is renewed or modified along with the knowledge of knowledge-cells.

\section{2 Basic actions}

Figure 9 describes the basic action of the system. The boxes shown in Fig. 9 represent various processes to be explained below.

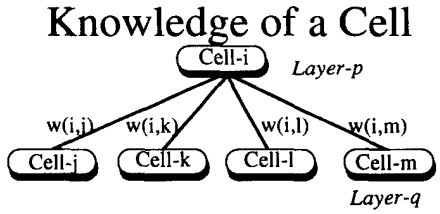

Connection weight: A positive number assigned to a connection between knowledge-cells $w(i, j), w(i, k), w(i, l), w(i, m)$

Connection intensity: A normalized connection weight

$w(i, j) / W, w(i, k) / W, w(i, l) / W, w(i, m) / w$

$\mathrm{W}=\mathrm{w}(\mathrm{i}, \mathrm{j})+\mathrm{w}(\mathrm{i}, \mathrm{k})+\mathrm{w}(\mathrm{i}, \mathrm{l})+\mathrm{w}(\mathrm{i}, \mathrm{m})$

\section{Knowledge: A vector composed of connection intensity}

Cell-i[ w(i,j)/W, w(i,k)/w, w(i,l)/W, w(i,m)/w ]

Fig. 8 Knowledge cells and their knowledge

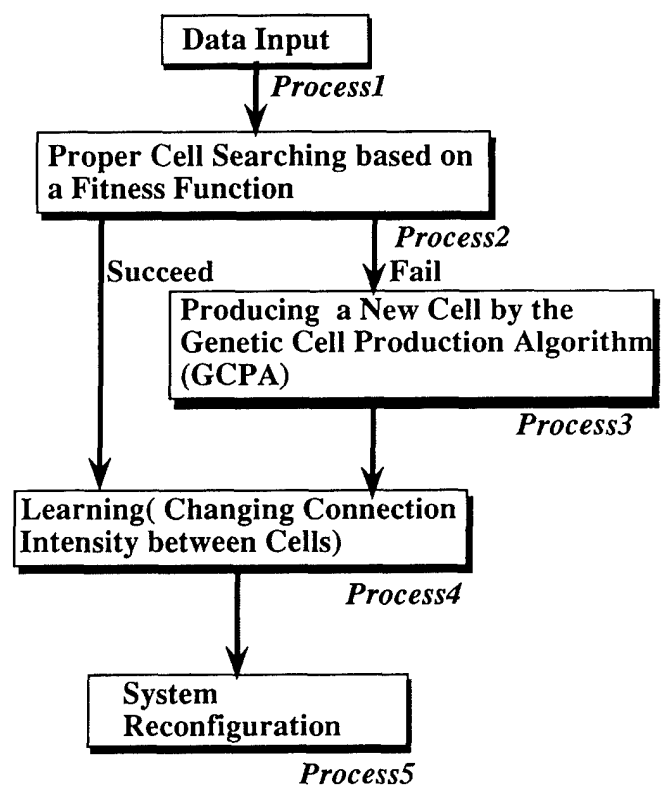

Fig. 9 Basic Motion of task planning system composed of knowledge-cells

Process 1: Calculation of correctness Correctness is defined by Eq. (1).

$$
C_{p}(i)=\sum_{k=1}^{m}\left\{\sigma(i, k) C_{p}(k)\right\}
$$

where $C_{p}(i)$ and $C_{q}(k)$ are correctness of cell $(i)$ of layer $p$ and that of cell $(k)$ of layer $q$, respectively, $\sigma(i, k)$ is the connection intensity between cell $(i)$ and cell $(k)$, and $m$ represents the number of cells in the lower layers which are connected with cell $(i)$. Correctness ranges in value from 0 to 1 , and represents the degree of compatibility between input data and cells. As based on Eq. (1), if the correctness of all the cells connected with cell $(i)$ is 1 , then the correctness of cell $(i)$ is also 1 , and the probability that cell $(i)$ is employed is high. After the correctness of a number of knowledge-cells is set equal to 1 as input data, the correctness of all the cells is computed based on their 
initial values and Eq. (1)

Process 2: Selection of cells

After the correctness of all the cells is computed, cell selection is carried out considering the values of correctness. As an example of selection, cells with higher correctness may be chosen ${ }^{6}$. If the system has a subsystem which is capable of executing a given task, then process 4 is carried out ; otherwise, process 3 is executed.

Process 3: Production of new cells When there are no cells or groups of cells (modules) which satisfy given conditions, then new knowledge cells are generated. The production of new cells is based on a genetic cell production algorithm which uses natural selection by living organisms as a model (refer to the next section).

Process 4: Changing connection weights between cells

If a cell is selected, the connection intensities between cells increase and the cell is selected more easily when the same demand is imposed on the system. Otherwise, connections of unemployed cells weaken.

Process 5: Reconfiguration of cell number and module number

In order to efficiently search for and produce cells and modules which satisfy the next demand, connections between cells may be changed. Grouping of cells having similar abilities is necessary to search for or produce in a few steps cells which satisfy the demand.

\section{Genetic Cell Production Algorithm}

In the models shown in Fig. 7, connections between cells are changed or the numbers of cells and layers are reconfigured. When the system does not contain a subsystem which satisfies the environment or a given task, then it is necessary to produce a new subsystem. In this section, a genetic cell production algorithm (to be abbreviated below as GCPA) will be proposed which incorporates a genetic algorithm (GA) method that uses genetics and evolution of living organisms as models in the process of new cell production.

GA is one of the search methods applied in recent years towards the optimization problem in combinations and others, and its basic actions are shown below $^{(8),(9)}$ :

1: Coding of objects according to genes. (Coding)

2: Fitness functions computed from various code rows are established and calculated.

3: Genes (code rows) with higher fitness functions are selected. (Reproduction)

4: Crossover between selected genes is initiated. (Crossover)

5: Codes are changed with a certain probability.
(Mutation) (Fig. 10)

The basic concept of GA is that among these steps, steps 3,4 and 5 are grouped as a generation and are carried out over many generations to obtain genes with higher fitness functions. Thus, GA can be said to model the survival of the fittest in a system of living organisms. In a similar way, CEBOT must adapt itself to differently changing unknown tasks and environments. For this reason, the concept of GA is applied to the new cell generation in the systems as shown in Fig. 11 and a new genetic cell production algorithm is proposed. The GCPA proposed here cousists of the procedures as follow:

1. Features possessed by cells (such as functions and knowledge) as genes are matched. (Coding)

2. The evaluation function possessed by each knowledge cell is calculated. (Fitness function)

3. A new knowledge cell candidate 1 is selected according to the state amount owned by cells. (Reproduction-1)

4. A candidate 2 is selected according to state amounts. (Reproduction-2)

5. A cross-over position is randomly determined, the features of cells are crossed over, and candidate 1 is expected to have the new features after cross-over is operated. (Crossover)

As an example, GCPA was applied to the model defined in the previous section (Fig. 6 and 7). In GCPA, a gene and its fitness function must be

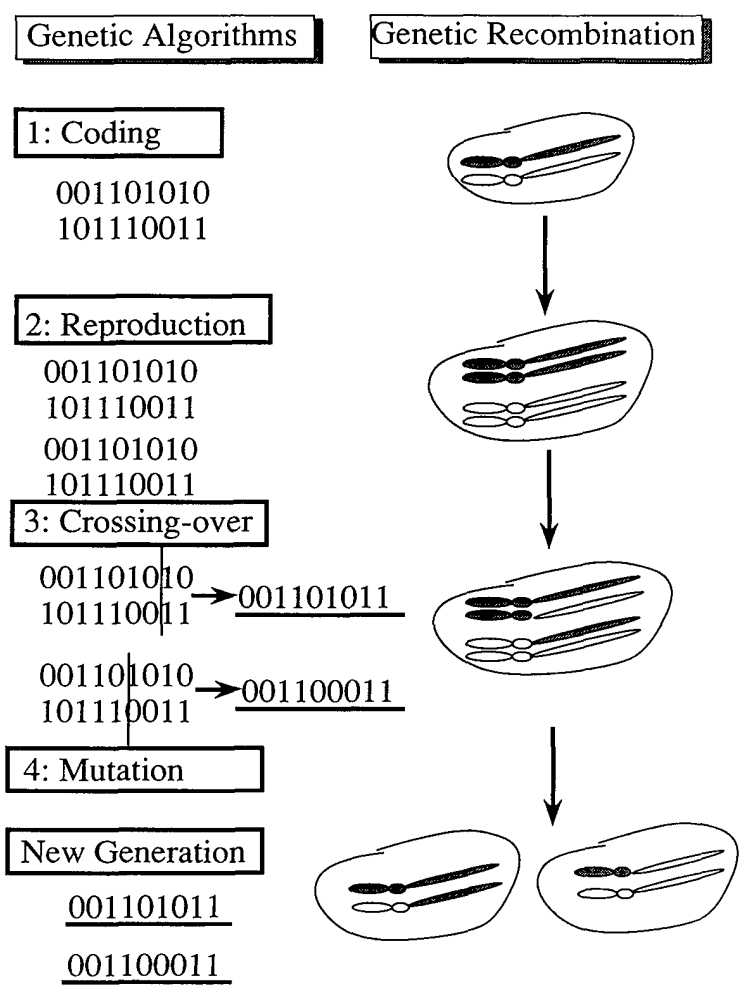

Fig. 10 Concept of genetic algorithms 


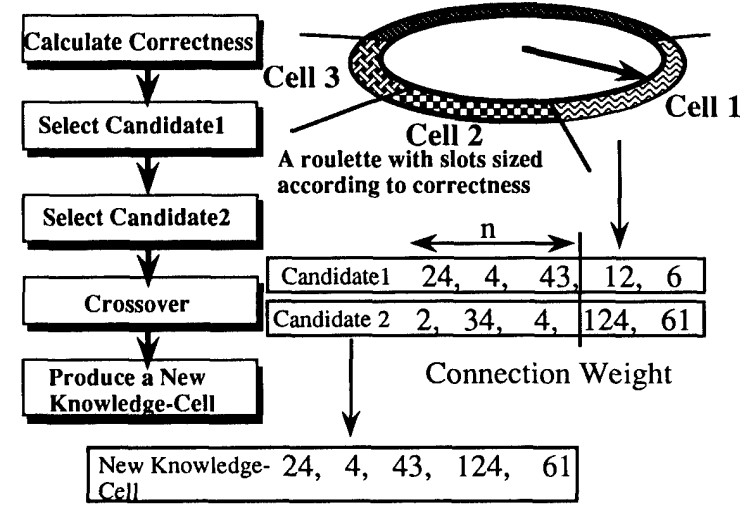

Fig. 11 Genetic cell production algorithms

determined. In this example, the knowledge of the cell clefined by Fig. 8 is used as a gene and the correctness defined by Eq. ( 1 ) is used as the fitness function. Moreover, Table 1 shows relationship among GA, GCPA and a living system.

\section{Experiments}

The distributed system under discussion in this paper has structures as shown in Fig. 6. Here, each cell can be considered as a subsystem for processing a certain sub-task, and more sophisticated tasks are processed by combining such cells efficiently. In order to verify efficient combinations of cells and generating actions, therefore, experiments were conducted, as an example to show a system behavior, by using the experimental equipment as described follow.

\section{1 System composition of experimental equip- ment}

The equipment for experiments is composed of a "robot manipulator" which handles tasks and a "task board" in which tasks are set by a computer (Fig. 12). The robot manipulator has a 6 -axle force torque sensor mounted on its end effector. The task-board consists of 9 numbered joysticks, and each joystick can move in four directions, namely up, down, left, or right. Both the manipulator and the task-board are connected to a computer, and the computer imbeds certain tasks in the task-board (Fig. 13). In these experiments, the task is defined as follows.

Task : to move certain numbered joy-sticks in certain directions in a certain determined order.

An example of the task given here is "to move \# 2 joystick right at step 1 and move \# 5 upward at step 2". Moreover, although the manipulator is controlled by the same computer, what type of task is given is not communicated at all. Also, the manipulator recognizes the end of the task by receiving a task termination signal from the computer.
Table 1 Comparison among GA, GCPA and natural world

\begin{tabular}{|c|c|c|}
\hline Natural & GA & GCPA \\
\hline gene & character & connection weight \\
\hline chromosome & string & knowledge \\
\hline \multirow[t]{2}{*}{ phenotype } & $\begin{array}{l}\text { parameter set } \\
\text { (quantity of systems) }\end{array}$ & $\begin{array}{l}\text { ability of a system } \\
\text { (quality of systems) }\end{array}$ \\
\hline & fitness function & correctness \\
\hline
\end{tabular}

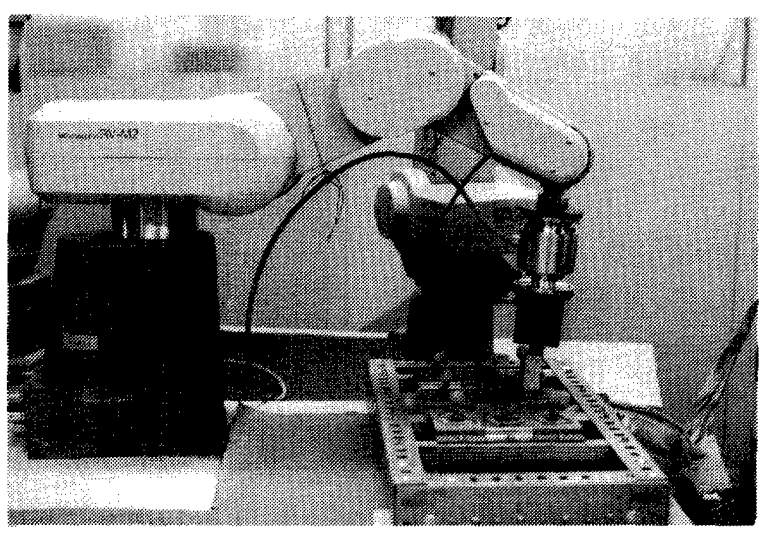

Fig. 12 Appearance of the experimental system

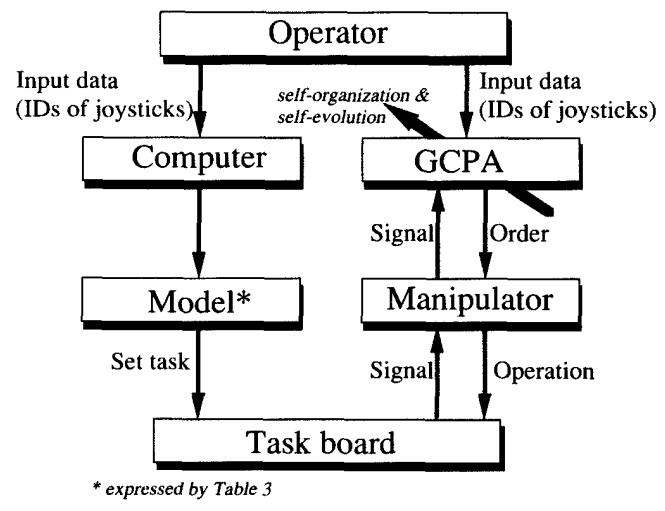

Fig. 13 Relationships among experimental equipment, operator and knowledge system (GCPA)

\section{2 Correspondent relations between distributed knowledge system and experimental equip- ment}

In the present experiments, new knowledge cells were produced and connection intensities are varied according to the algorithm shown in section 5 while the task given to the task-board was carried out. Here, the task is expressed as a combination of a ID of joystick and the direction of the joystick operation. These task descriptive methods and data structures have already been published ${ }^{6}$. It is assumed here that the addresses and operational directions of joysticks are selected as subsystems and correspond to the cells shown in Fig. 7, and combinations of joysticks and their operational directions are described by sets of those cells. In the present experiments, a four-layered 
Table 2 Correspondence between respective layers of knowledge-cell and experimental equipment

\begin{tabular}{|c|c|}
\hline Knowledge Cells & Experimental Equipment \\
\hline Layer-4 & Complicated Tasks \\
\hline Layer-3 & Given Tasks \\
\hline Layer-2 & Address of Joy-sticks \\
\hline Layer-1 & OperationalDirection \\
\hline
\end{tabular}

Table 3 Values of $G_{i j}$ and $H_{i}$ set at task board

\begin{tabular}{|c|l|l|l|l|l|l|l|}
\hline $\mathrm{H} 1$ & $\mathrm{H} 2$ & $\mathrm{H} 3$ & $\mathrm{H} 4$ & & & & \\
\hline 7 & 84 & 29 & 31 & & & & \\
\hline G11 & $\mathrm{G} 12$ & $\mathrm{G} 13$ & $\mathrm{G} 14$ & $\mathrm{G} 31$ & $\mathrm{G} 32$ & $\mathrm{G} 33$ & $\mathrm{G} 34$ \\
\hline 0.871 & 0.043 & 0.043 & 0.043 & 0.043 & 0.043 & 0.871 & 0.043 \\
\hline $\mathrm{G} 21$ & $\mathrm{G} 22$ & $\mathrm{G} 23$ & $\mathrm{G} 24$ & $\mathrm{G} 41$ & $\mathrm{G} 42$ & $\mathrm{G} 43$ & $\mathrm{G} 44$ \\
\hline 0.043 & 0.871 & 0.043 & 0.043 & 0.043 & 0.043 & 0.043 & 0.871 \\
\hline
\end{tabular}

structure is considered as a special case of a system defined and modeled in Fig. 7. The layers are called the first to the fourth layer from the lowest upward. The tasks are described by the operational directions and numbers of the joysticks, the first layer (layer-1) of known knowledge cells is used as the layer for describing moving directions, and a second layer (layer-2) is formed above the first layer and used as the layer for expressing the numbers of the joysticks. Tasks formed by combinations of these are assigned to cells in the third layer (layer-3) and sets of tasks to cells in the fourth layer (layer-4). Table 2 shows the relationships between the distributed knowledge system and the experimental equipment. Moreover, relationships among the manipulator, the task-board, and GCPA are shown in Fig. 13. The operator gives the address of the joystick to be operated to the computer and GCPA. The computer possesses a certain model described by $G_{i j}$ and $H_{i}$ defined in Table 3 and the next section, and generates a task by using this model and establishes it on the task-board. In the self-organizing system, the correctness of the cell corresponding to a given joystick is set equal to 1 , and an action row is generated according to the algorithm as shown in Figs. 9 and 11, and this action is carried out on the task-board by the use of the manipulator.

Signals are transmitted to the self-organizing system only in cases where the operation carried out by the manipulator is identical to the task established beforehand. The self-organizing system creates models within itself through a number of trials. At the initial stages of the experiments, the knowledge cells inside the self-organizing system are connected on an equal basis, and since the number of cells in the third and fourth layers is small, the task-board is operated randomly combining permutations for the purpose of generating operational rows, and the processing of the tasks takes time. However, it is possible to learn characteristics of the task-board by operating the task-board a number of times; for example, a correct solution is more easily obtained if joystick-1 is pushed down in the direction of 1 . Thus, the time taken for obtaining correct solutions (number of operations) can be expected to be gradually reduced. These points will be verified by the experiments indicated in the following sections.

\section{3 Experimental procedures}

The experiments were conducted by the following procedures.

STEP 1. The relative frequency $\left(G_{i j}\right)$, which indicate probability that operational direction $(j)$ of joystick $(i)$ is correct, is given.

STEP 2. A positive constant $\left(H_{i}\right)$ is assigned to each joystick $(i)$.

STEP 3. Correct tasks are set on the task-board.

A correct task is expressed in terms of which joystick should be moved in which direction and at which step. The correct direction of each joystick is $G_{i j}$, and the order of priority of joystick operations is then determined stochastically by using $H_{i}$. For example, in case $G_{i j}=0.8$, for joystick $(i)$ there is $80 \%$ probability that direction $j$ is chosen as a correct one. However, this does not always mean that it should be pushed down in direction $j$; there is $20 \%$ probability that other directions are correct. These correct directions are changed at each trial.

STEP 4. The robot manipulator is informed of the number of the joysticks to be moved for the execution of the task in question. This corresponds to Process 1 of Fig. 9.

STEP 5. Until the task is completed (until task terminating signals arrive from the computer), the manipulator keeps moving joy-sticks in various directions.

STEP 6. At the completion of the task, the manipulator carries out self-organization of knowledge for more efficient processing of tasks on the task-board by changing the knowledge structure and producing new knowledge cells. The procedures from Process 2 to Process 5 of Fig. 9 are carried out.

\section{STEP 7. Return to STEP 3.}

All these procedures are carried out, and the total number of joystick operations (to be called operation time) is recorded.

\section{4 Experimental results}

Experiments were carried out according to the method described in the previous section, and the time for task execution was recorded and plotted in Fig. 14. The time for task execution is corresponded to a 


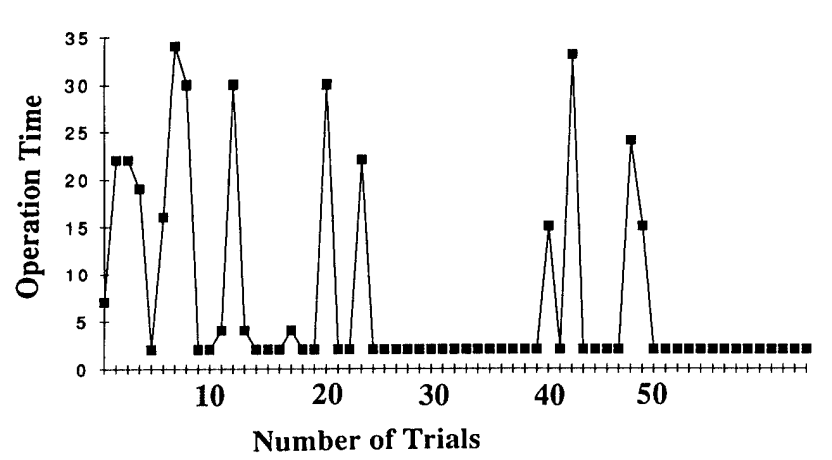

Fig. 14 Results of experiments

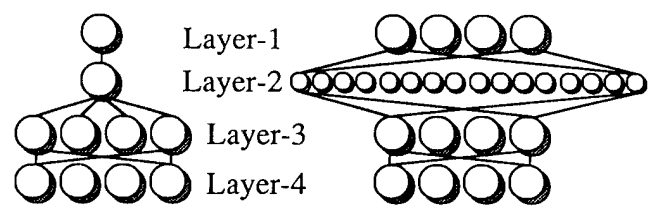

(a) Initial State

(b) Final State

Fig. 15 Change in number of cells contained in each layer at initial and final conditions of experiment

number of actions of the manipulator between a time when the manipulator received task from a computer in Fig. 13 and a time when the manipulator received the task termination signals from the computer. Figure 14 shows the number of trials on the horizontal axis and operation times on the vertical axis. When the number of trials is small, the characteristics of the task board have not been learned and joysticks are operated randomly so that the operation times are high. As the number of trials increases, that of knowledge cells increases, and both tight and weak relationships are formed among knowledge-cells. This is equivalent to the fact that each joystick on the task-board is closely related to the operational directions that are apt to be correct. As the number of trials increase, the operation times are reduced because the task-board is operated by using the empirical knowledge.

The jumps on the number of trials in Fig. 14 represent cases where low probabilities of $G_{i j}$ in Table 3 were chosen as correct tasks and indicate times when new cells are being generated. The operation times at the time of new cell generation tend to decrease, which implies that this system has learned the characteristics of the task-board through empirical learning and produces knowledge-cells more efficiently. Moreover, the number of cells in each layer is different as shown in Fig. 15, which shows an example of software self-organization of CEBOT based on the GCPA. The system creates knowledgecells to make the total system adapt to the given tasks and environment.

\section{Conclusions}

This paper reported the self-organization and self-evolution of a dynamically reconfigurable system from the viewpoint that a system must possess selforganization and self-evolution if it is to adapt itself to unknown environments and tasks. In order for a system composed of sets of subsystems to adapt itself efficiently to unknown environments and tasks, the generation and deletion of new subsystems are necessary, and a genetic cell production algorithm was proposed which is an application of a genetic algorithm having as its basic principle the modeling of natural selection in the world of living organisms. This algorithm was then applied to a task planning system of a cellular robot, a form of a dynamically reconfigurable robotic system, and its effectiveness was verified by experiments in which during the operation of unknown objects, models of those objects are self-organized internally. Thus, this system is considered to be self-evolving, because the system generates subsystems by using an algorithm modeled after a gene of a living organism and also because the system as a whole improves efficiency by changing the connections among subsystems.

\section{References}

(1) Fukuda, T. and Nakagawa, S., A Dynamically Reconfigurable Robotic System (Concept of a System and Optimal Configuration), Proc. IECON '87, (1987), p. 588.

(2) Fukuda, T. and Nakagawa, S., Approach to the Dynamically Reconfigurable Robotic System, Journal of Intelligent and Robotic Systems, Vol. 1, (1988), p. 55

(3) Fukuda, T., Nakagawa, S., Kawauchi, Y. and Buss, M., Self-Organizing Robots Based on Cell Structures CEBOT, Proc. of IEEE Int'1 Workshop on Intelligent Robots and Systems (IROS'88), (1988), p. 145.

(4) Fukuda, T. and Kawauchi, Y., Cellular Robotic System (CEBOT) as One of the Realizations of Self-Organizing Intelligent Universal Manipulator, Proc. of IEEE Int'1 Conf. on Robotics and Automation' 90 (R\&A'90), (1990), p. 662.

(5) Fukuda, T. and Kawauchi, Y., Communication and Distributed Intelligence for Cellular Robotic System (CEBOT), Proc. of 1990 JAPAN-U. S. A. Symposium on Flexible Automation, (1990), p. 1085.

(6) Fukuda, T., Kawauchi, Y. and Hara, F., Distributed Intelligence System Based on Knowledge Modules of Cellular Robotic System (CEBOT), Proc. of IFAC Int'1 Conf. on Distributed Intelligence System 91 (DIS 91), (1991), p. 195.

( 7 ) Fukuda, T., Ueyama, T., Kawauchi, Y. and Arai, F., Concept of Cellular Robotic System (CEBOT) 
and Basic Strategies for Its Realization, Computer Elect. Eng'g Vol. 18, No. 1, (1992), p. 11.

(8) Grefenstette, J.J., Gopal, R., Rosmalta, B. and Van Gucht., D., Genetic Algorithms for the Traveling Salesman Problems, Proc. of the 1st
Int'l Conf. on Genetic Algorithms and their Applications, Grefenstette, J.J. (editor), (1985).

(9) Goldberg, D.E., Genetic Algorithms in Search Optimization, and Machine Learning, (1989), Addison-Wesley Publishing Company Inc. 